\title{
EFFECT OF DYEING ON WRINKLE PROPERTIES OF COTTON CROSS-LINKED BY BUTANE TETRACARBOXYLIC ACID (BTCA) IN PRESENCE OF TITANIUM DIOXIDE $\left(\mathrm{TIO}_{2}\right)$ NANOPARTICLES
}

\author{
Emadaldin Hezavehi*1, Sheila Shahidi ${ }^{1}$, Peyman Zolgharnein ${ }^{1,2}$ \\ ${ }^{1}$ Department of textile, Faculty of Engineering, Arak branch, Islamic Azad University, Arak, Iran \\ E-mail: Emadhezavehi@yahoo.com, E-hezave@iau-arak.ac.ir, Shahidish@yahoo.com, \\ ${ }^{2}$ The University of Sheffield, Material Science and Engineering Department, Sheffield, United Kingdom, S3 7HQ \\ E-mail: Pzolgharnein2@sheffield.ac.uk
}

\begin{abstract}
:
This study evaluates the wrinkle behaviour and wrinkle resistant properties of cotton fabrics dyed by Direct Blue $2 B$ in the presence and absence of nano-TiO ${ }_{2}$ particles. A finishing process on samples was performed before dyeing by means of 1,2,3,4-butanetetracarboxylic acid (BTCA) and sodium hypophosphite (SHP) using a pad dry cure method. Such experiments as wrinkle recovery test (AATCC-128), water drop test and dye fastness determination (ISO 105-C01) were conducted to understand how current treatment impacted the cotton fabrics' properties. Finally, samples were characterised using the $X$-ray diffractometery method to scrutinise how crystallinity changes and dominates the structural parameter (wrinkle behaviour) of cotton fabrics. Furthermore, scanning electron microscopy (SEM) was used to visualise the surface variations of the samples before and after dyeing process. The results showed that the wrinkle resistant of cross-linked fabrics have been improved after direct dyeing. Furthermore, levelness dyeing and good fastness properties of dyes on cross-linked fabrics were observed.
\end{abstract}

\section{Keywords:}

Wrinkle properties, dyeing, cotton fabrics, 1,2,3,4-butanetetracarboxylic (BTCA), Sodium hypophosphite (SHP), Nano-Titanium Dioxide $\left(\mathrm{TiO}_{2}\right)$

\section{Introduction}

Cotton is a well-known cellulosic fibre chemically linked by many hydroxyl groups. Textiles made from cellulose get usually finished in order to improve their properties, where dimensional stability and crease resistance are anticipated. These might be more desirable properties if cross-linking agents, which react with hydroxyl groups of cellulose fibres, exist on the structure. The newer approach for producing non-swelling or durable press cellulose fabric is making use of the multifunctional cross-linking agents where 1,2,3,4-buthanetetracarboxylic acid (BTCA) provides an alternative possibility for non-formaldehyde crease-resistant finishing [1-8].

Today, using nanostructure materials ( 1 to $100 \mathrm{~nm}$ in dimension) for developing desired textile characteristics and enhancing its attributes are becoming widespread in potential applications. Nanoparticles have a large surface area-tovolume as well as high surface energy, i.e. better affinity for textile substrates thereby leading to an increase in durability while maintaining breathability and/or hand feel. Very recent studies have discussed the application of nano-sized $\mathrm{TiO}_{2}$ in textile finishing using the sol-gel technique to impart effective protection against UV radiation and bacteria, as well as selfcleaning due to its photocatalytic activity [2, 9-17]. Titanium dioxide $\left(\mathrm{TiO}_{2}\right)$ or nano- $\mathrm{TiO}_{2}$ as a catalyst or co-catalyst has been also utilised to enhance crease recovery property of cotton fabric and wrinkle properties of cotton/polyester blended Fabric $[18,19,25]$. As a heterogeneous catalyst, it is added as a co-catalyst to maximise finishing performance and minimise the side effect. It is sometimes called surface catalyst as the reaction involves the catalyst to provide its surface on which the reactants or substrates temporarily get adsorbed. The bonds in the substrate become sufficiently weakened for the new bonds to be composed. Since bonds between product and catalyst are weaker, the products may be released easily. Metal oxide is very stable towards heat and pressure, and so it can be used at relatively high temperature. The effect of catalyst size, which is known as 'size effect', is a prominent characteristic for recognising the fundamental catalytic properties. Ultrafine catalyst is often more active as compared with larger ones probably due to their high surface area. Therefore, small catalytic particle size can stimulate the chemical reactivity.

The major undesirable properties of cotton fabric are shrinkage and wrinkling [20]. The wrinkle-resistant property of cotton specimens treated by BTCA and catalysed by SHP in the presence of $\mathrm{TiO}_{2}$ or nano- $\mathrm{TiO}_{2}$ has been evaluated elsewhere $[20,25]$. Different concentrations of $\mathrm{TiO}_{2}$ or nano- $\mathrm{TiO}_{2}$ used in the wrinkle resistant finishing process have been experimented in order to determine their optimum concentrations [20-23]. There will defiantly be finishing and dying process influences on the fabrics' hand and their overall performance. In the finishing process, internal stresses imposed during spinning and weaving get eliminated, and fabrics become almost relaxed sometimes even complete relaxation may occur. In a complex scenario, using different finishing and dyeing substances and treatments can make a considerable difference in end products. There is no experienced evidence to shed light on dyeability of fabrics after using the treatments, and any possible chemical interaction with 
structural properties (especially wrinkle-resistant properties of samples being under exhaustive finishing and dying process) of the cotton fabrics. Meanwhile, the question about wrinkle properties of cotton fabrics in the presence and absence of pigments along with the finishing treatment is still unanswered and draws attention to perform further experiments. Dyeing effects on wrinkle resistant properties remain an aspect that need to be addressed before commercialising the products.

In this research work, the wrinkle resistant property of cotton fabrics treated by BTCA, SHP in both presence and absence of nano- $\mathrm{TiO}_{2}$, before and after dyeing will be investigated. Along the way, dyeability of treated fabrics is another object of consideration.

\section{Experimental}

\subsection{Materials}

$100 \%$ plain woven cotton fabric (density per cm: $25 \times 20$; yarn count $20 \times 30 \mathrm{Ne}$ ) with the weight of $136\left(\mathrm{~g} / \mathrm{m}^{2}\right)$ and size of $20 \times 20 \mathrm{~cm}^{2}$ were used. The cross-linking agent was applied such a 1,2,3,4-butanetetracarboxylic acid (BTCA) supplied by Merck company, Germany. The sodium hypophosphite (SHP) was utilised as catalyst supplied by Fluka Company, Swiss and Nano- $\mathrm{TiO}_{2}$ as a co-catalyst supplied by Degussa Chemical Company, Germany.

\subsection{Pad-dry-cure treatment}

Pad dry-cure method was employed for treating the cotton fabric samples. Different concentrations of nano- $\mathrm{TiO}_{2}$ solutions along with wrinkle-resistant finishing agents were prepared according to the stated conditions in Table 1.

The fabric samples were dipped and padded with $100 \mathrm{ml}$ of each solution until the wet pick up of $80 \%$ was achieved at room temperature. The specimens were then dried at $85^{\circ} \mathrm{C}$ for 5 minutes and cured at $170^{\circ} \mathrm{C}$ for $2 \mathrm{~min}$. Finally, the fabrics were conditioned at $21 \pm 1^{\circ} \mathrm{C}$ and $65 \pm 5 \%$ relative humidity $(\mathrm{RH})$ for 24 hours prior to any treatment. It should be noted that in sample $C$, padding was performed with BTCA and SHP then

Table 1. Identification of samples.

\begin{tabular}{|c|c|c|c|c|}
\hline \multirow[b]{2}{*}{ Sample } & \multirow[b]{2}{*}{$\begin{array}{l}\text { Order of finishing } \\
\text { agents }\end{array}$} & \multicolumn{3}{|c|}{ Composition } \\
\hline & & $\begin{array}{c}\text { BTCA } \\
\text { (wt/wt } \\
\%)\end{array}$ & $\begin{array}{c}\text { SHP } \\
\text { (wt/wt } \\
\%)\end{array}$ & $\begin{array}{c}\text { TiO2 } \\
\text { (wt/wt } \\
\% \text { ) }\end{array}$ \\
\hline$A$ & BTCA + SHP & 6 & 6 & - \\
\hline $\mathrm{B}$ & $\mathrm{TiO}_{2}$ & - & - & 2 \\
\hline $\mathrm{C}$ & $\mathrm{BTCA}+\mathrm{SHP} \gg>\mathrm{TiO}_{2}$ & 6 & 6 & 1 \\
\hline $\mathrm{D}$ & $\mathrm{TiO}_{2} \gg>\mathrm{BTCA}+\mathrm{SHP}$ & 6 & 6 & 1 \\
\hline$E$ & $\mathrm{BTCA}+\mathrm{SHP}+\mathrm{TiO}_{2}$ & 6 & 6 & 1 \\
\hline $\mathrm{F}$ & - & - & - & - \\
\hline
\end{tabular}

with $\mathrm{TiO}_{2}$ solution was performed. However in case of sample $\mathrm{D}$, padding was initiated by $\mathrm{TiO}_{2}$ followed by BTCA and SHP. Simultaneous treatment with $\mathrm{TiO}_{2}, \mathrm{BTCA}$ and SHP was carried out for sample E. Sample F was untreated cotton without any cross-linking agents.

\subsection{Dyeing procedure and evaluation}

The treated and untreated fabrics were dyed using Direct Blue 2B (Cl. Direct Blue 6). Dye-baths were prepared by dissolving $1 \mathrm{wt} \%$ dye in distilled water and the temperature was raised to $45^{\circ} \mathrm{C}$. Afterwards, fabric samples were set to the dye-baths. For conventional dyeing of untreated cotton, $30 \mathrm{~g} / \mathrm{L}$ of sodium chloride was added to the dye-baths. The above mentioned temperature was maintained for $10 \mathrm{~min}$, and then was gradually increased to $80^{\circ} \mathrm{C}$ with $2^{\circ} \mathrm{C} / \mathrm{min}$ rate and dyeing continued for $40 \mathrm{~min}$.

After dyeing, the cotton fabrics were removed from the dyebaths and rinsed thoroughly with tap water. Then the dyed fabric was subjected to boiling in a solution containing $2 \mathrm{~g} / \mathrm{L}$ anionic detergent at a liquor-to-goods ratio of $25: 1$ for $15 \mathrm{~min}$ until no dye was removed off, and then rinsed and allowed to air dry.

Colour intensities of the dyed fabrics along with reflection factor $(R)$ were measured using a UV VIS-NIR Reflective Spectrophotometer, over the range of $200-800 \mathrm{~nm}$. The relative colour strength (K/S value) was then established according to the Kubelka Munk equation (1), where $K$ and $S$ stand for the absorption and scattering coefficient, respectively:

$$
\frac{K}{s}=\frac{(1-R)^{2}}{2 R}
$$

\subsection{Wrinkle recovery and water drops tests}

The wrinkle recovery properties of specimens were measured applying wrinkle recovery tester AATCC-128 [22,24,26]. The wettability of the samples before and after dyeing was evaluated by measuring the time of adsorption of 4 distilled water drops through the fabrics.

\subsection{Determination of dye fastness}

The wash-fastness properties of the samples were measured according to the ISO 105-C01 standard. The colour hue variation of the fabric and degree of staining on the adjacent fabrics was measured after drying. For light-fastness measurements, the fabrics were exposed to daylight for 2 and 7 days according to the daylight ISO 105-B01, and the changes in the colour (fading) were assessed by the blue scale. Also Electronic Crockmeter/Rubbing Fastness Tester AATCC 8 was used to determine the colour fastness of textiles among dry or wet rubbing.

\subsection{X-ray diffraction and microscopical evaluation}

The percentage of crystallinity of the samples, size of crystals and also distance between them was determined by SEIFERT (PST-3003) X-ray diffractometer. A scanning electron 
microscope (SEM) XL30, made by the Dutch Phillips Company, was used to evaluate the fabrics' structural morphology before and after dyeing.

\section{Results and discussion}

In the current study, as it was mentioned earlier, effect of dyeing on wrinkle resistant properties of cross-linked fabrics in both the presence and absence of nano- $\mathrm{TiO}_{2}$ is investigated. The results are shown in Table II. As can be seen, the addition of BTCA could enhance significantly the wrinkle-resistance of cotton fabrics, especially in the presence of SHP and nano$\mathrm{TiO}_{2}$ which acted as a co-catalyst. This result complies with previously reported achievement [20]. Generally, during the curing process, the BTCA reacts with the cellulose molecules of cotton fabric most probably through the formation of cyclic anhydrides as reactive intermediates, which in turn, esterifies the cotton cellulose. In the presence of SHP, the BTCA molecules are able to crosslink some hydroxyl groups of cellulosic macromolecules effectively by accelerating the formation of anhydrides from BTCA, contributing to greater wrinkle recovery angle (WRA). When the nano- $\mathrm{TiO}_{2}$ particles were added in the BTCA treatment, the particles probably restricted the molecular movement of cellulose, and the BTCA molecules were even catalysed, leading to the enhancement of WRA of tested specimens. The particles enhanced the catalytic reaction towards the faster formation of an ester bond between the cyclic anhydride ring and the hydroxyl group of cellulose. However, it should be explained that adding $\mathrm{TiO}_{2}$ in absence of BTCA causes better wrinkle resistant property, but is not considerable.

There are two theories for the mechanism of anti-wrinkle finishing of cotton fabrics. The deposition theory proposes that small particles of finishing agents are dispersed in amorphous region of the fibres where they might be deposited after resinification and form $\mathrm{H}$-bonds with the fibre molecules. This may restrict the movement of the molecular chains and decrease deformation of the fabrics. The cross-linking theory proposes that finishing agents form covalent bonds with fibre molecules, and connect the adjacent molecular chains within the fibres. This gives the recoverability enhancement of the deformed fibres and increases the wrinkle resistance.

Also, as can be observed, after dyeing, the wrinkle resistant properties of cotton fabrics has been improved significantly (Table 2). This improvement was mainly attributed to the fact that the direct dye could react with cellulose fibres through their hydrogen bonds, which led to the cross-linking of cellulosic fibres and limited the relative movement of the molecular chains. It was found that both aforementioned theories are extensible for the wrinkle resistant properties of dyed treated cotton fabrics.

The results related to wettability of samples before and after dyeing are shown in Table 3 . It is depicted that immediately after putting droplets on the surface of untreated and BTCA/ SHP treated fabrics, they were absorbed deeply. In contrast, by adding nano- $\mathrm{TiO}_{2}$, results were changed and longer absorption time has been observed. Existence of nano- $\mathrm{TiO}_{2}$ particles provides water repellent property on the surface. Since nanoparticles can block some of the hydrogen bonds of cellulosic chains, the absorption time could be increased. However, it should be mentioned that Ultraviolet light plays an important role on nano- $\mathrm{TiO}_{2}$ properties. It may imply the photocatalytic activity of nano- $\mathrm{TiO}_{2}$, particularly, in this research work.

Moreover, the presence of BTCA causes more absorption of nano- $\mathrm{TiO}_{2}$ on the surface of fibres. It is seen that the water absorption on $\mathrm{BTCA} / \mathrm{SHP} / \mathrm{TiO}_{2}$ treated sample takes a longer time rather than that for $\mathrm{TiO}_{2}$ treated cotton. Furthermore, the order of padding first by $\mathrm{BTCA}$ or $\mathrm{TiO}_{2}$ may not give the considerable variation on the surface properties.

After direct dyeing, increasing the time for water absorption can be attained. It is due to the fact that dye particles might be absorbed by hydroxyl groups of cellulose chains and carboxyl groups of BTCA and some of them make some complexes with titanium dioxide nano-particles. Therefore, the hydroxyl groups of cellulose available to absorb water are reduced and more water repellent effect appears on the surface of cotton samples.

Table 2. The wrinkle resistant of samples before and after dyeing with Direct dye.

\begin{tabular}{|c|c|c|}
\hline samples & $\begin{array}{c}\text { before dyeing } \\
\text { (REPLICA) }\end{array}$ & $\begin{array}{c}\text { after dyeing } \\
\text { (REPLICA) }\end{array}$ \\
\hline A & 2 & 3 \\
\hline B & 2 & 3 \\
\hline C & 3 & 4 \\
\hline D & 3 & 4 \\
\hline E & 3 & 4 \\
\hline F & 1 & 2 \\
\hline
\end{tabular}

Table 3. Wettability (water absorption time) of samples before and after direct dyeing.

\begin{tabular}{|c|c|c|}
\hline samples & $\begin{array}{c}\text { before dyeing } \\
\text { (second) }\end{array}$ & $\begin{array}{c}\text { after dyeing } \\
\text { (second) }\end{array}$ \\
\hline A & 1 & 4 \\
\hline B & 10 & 11 \\
\hline C & 18 & 19 \\
\hline D & 20 & 25 \\
\hline E & 20 & 30 \\
\hline F & 1 & 2 \\
\hline
\end{tabular}

In the other point of view, the dyeability of finished fabrics is quite crucial. Therefore, this draws more attention the consideration of dyeability of cotton samples treated with different condition of $\mathrm{BTCA} / \mathrm{SHP} / \mathrm{TiO}_{2}$ in the current study. The results related to colour intensities of fabric specimens were considered using reflection spectrophotometry. These are illustrated in Figures 1 and 2 . It is clearly observable that the reflection factor for 


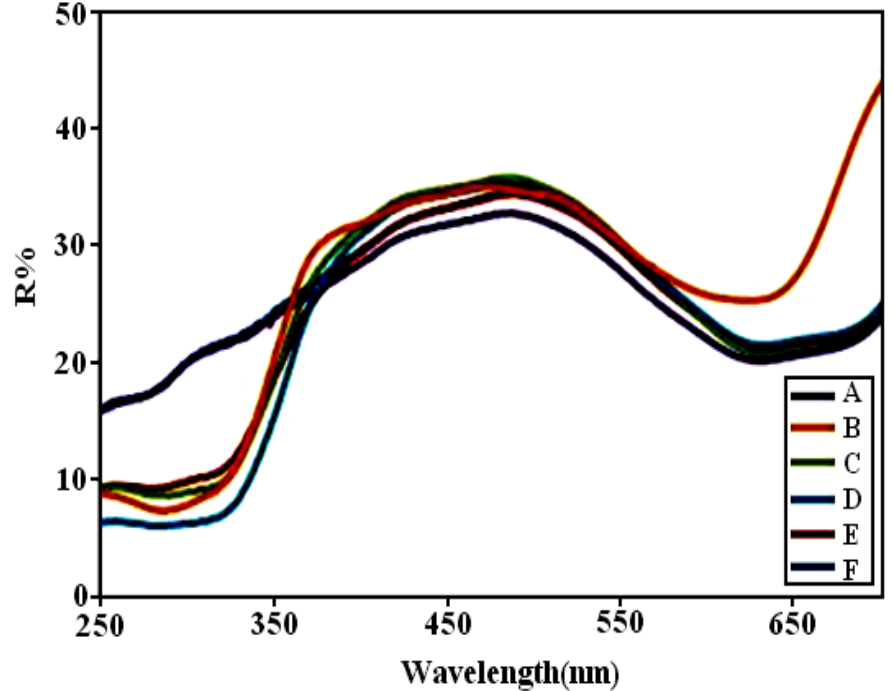

Figure 1. The reflection spectrophotometry of dyed samples in range of $250-700 \mathrm{~nm}$.

sample (A) that was treated just by BTCA/SHP is less than the others. It means that Sample (A) is somewhat darker. BTCA as a poly carboxylic acid, which has four carboxylic groups in its structure can have good potential for improving the dyeability of textile fabrics. These groups with attendance of remained and unblocked hydroxyl groups on the surface of cellulose can absorb more amount of direct dye. The reflection curves for samples $E$ and $F$ are overlapping. Also the rest of samples are covering each other. It means that the shade, hue and brightness of the samples will not change after treating with BTCA, SHP and $\mathrm{TiO}_{2}$. As the shade of used dye was blue, the adjusted wavelength is limited near $350-500$. The relative colour strength $(\mathrm{K} / \mathrm{S})$ of the samples was measured according to Kubelka Munk equation and results are shown in Figure 3. As expected, the amount of K/S for sample $A$ is more than the others. However for the rest of samples the K/S curves are overlapping and the results are very close to each other. As it can be seen, presence of nano- $\mathrm{TiO}_{2}$ as a finishing agent causes increasing the water absorption time on the samples, but its role as a co-catalyst becomes unremarkable on dye ability of the samples.

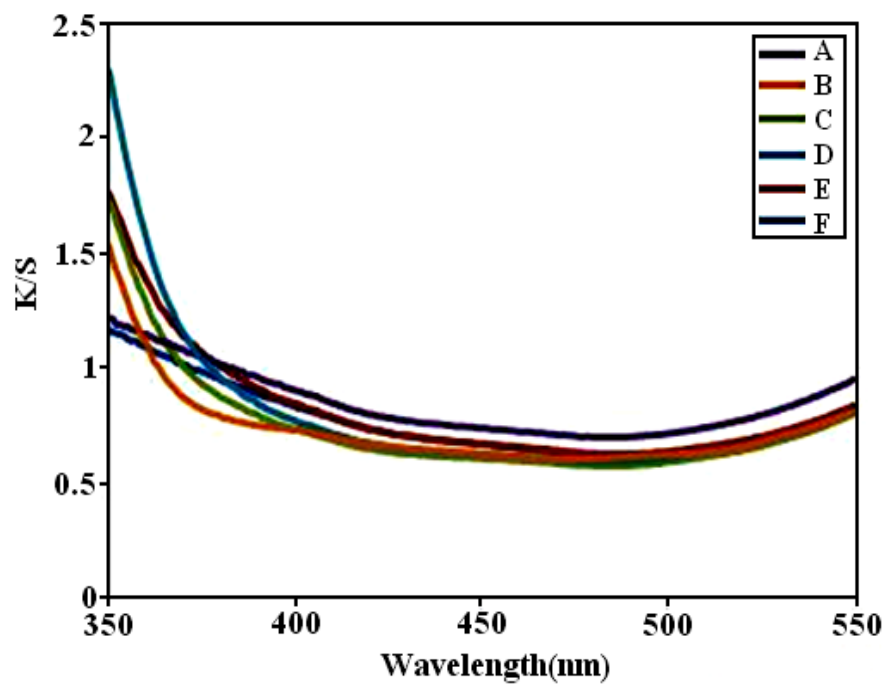

Figure 3. The relative colour strength for dyed samples.

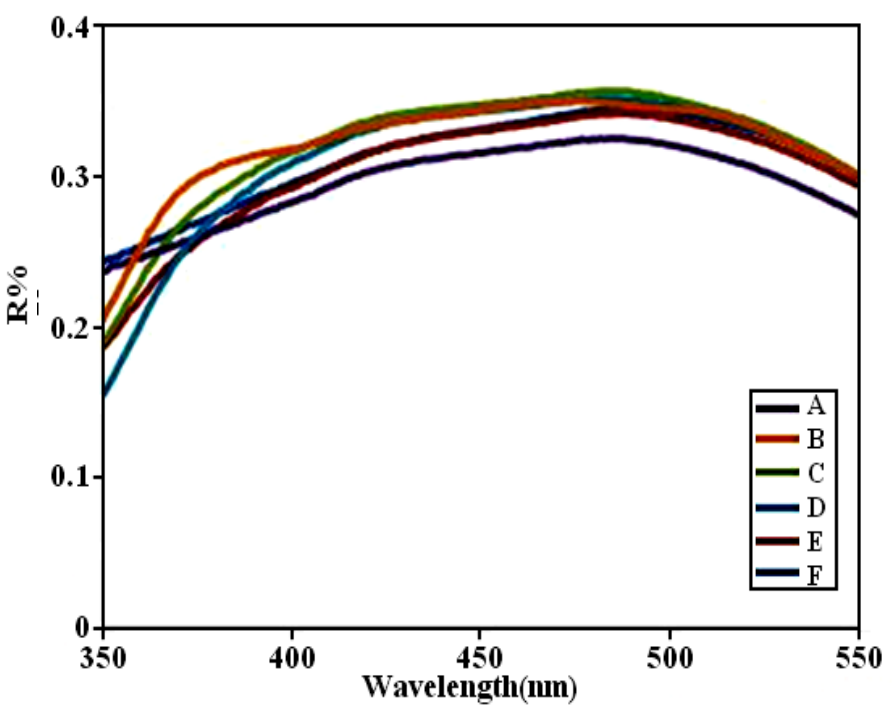

Figure 2. The reflection spectrophotometry of dyed samples in range of $350-550 \mathrm{~nm}$.

Nano- $-\mathrm{TiO}_{2}$ is a suitable chelating site for direct dyes. These nano-particles can make complex with dye and prepares larger molecule in space between and inside the fibres. It can be concluded that dye particles link to hydroxyl groups of cellulose chain and also make complex bonds with $\mathrm{TiO}_{2}$ nano-particles. Also as it can be seen in Table 4, the durability and fastness of dyed samples are satisfactory and even better than untreated dyed sample. Change of shade and staining of adjacent fabrics is higher than the values obtained on the untreated cotton. Dry and wet rub fastness of the dyes on the dyed fabrics was also comparable with that obtained for untreated dyed sample. Therefore, it could be induced that the direct dye could not only be applied to cross-linked cotton dyeing with satisfactory dyeing properties, but could also provide the cotton with excellent antiwrinkle properties.

Table 4. The fastness properties of dyed cross-linked and untreated samples.

\begin{tabular}{|c|c|c|c|c|}
\hline \multirow{2}{*}{ samples } & \multicolumn{2}{|c|}{$\begin{array}{c}\text { Wash } \\
\text { fastness }\end{array}$} & \multicolumn{2}{c|}{ Rubbing fastness } \\
\cline { 2 - 5 } & Changes & staining & Dry & Wet \\
\hline A & $4-5$ & 5 & $4-5$ & $4-5$ \\
\hline B & 4 & $4-5$ & $4-5$ & $4-5$ \\
\hline C & $4-5$ & $4-5$ & $4-5$ & $4-5$ \\
\hline D & $4-5$ & $4-5$ & $4-5$ & $4-5$ \\
\hline E & $4-5$ & $4-5$ & $4-5$ & $4-5$ \\
\hline F & $3-4$ & 4 & 4 & 4 \\
\hline
\end{tabular}

\subsection{X-ray diffraction analysis}

XRD is a crystal structure analysis method using the atomic arrays within the crystals as a three-dimensional grating to diffract a monochromatic beam of X-rays. The angles at which the beam is diffracted are used to calculate the inter-planer atomic spacing (d-spacing) giving information about how the 
atoms are arranged within the crystalline compounds. X-ray diffraction is also used to measure the nature of polymer and extent of crystalline present in the sample. The results of XRD analysis are illustrated in Figure 4.
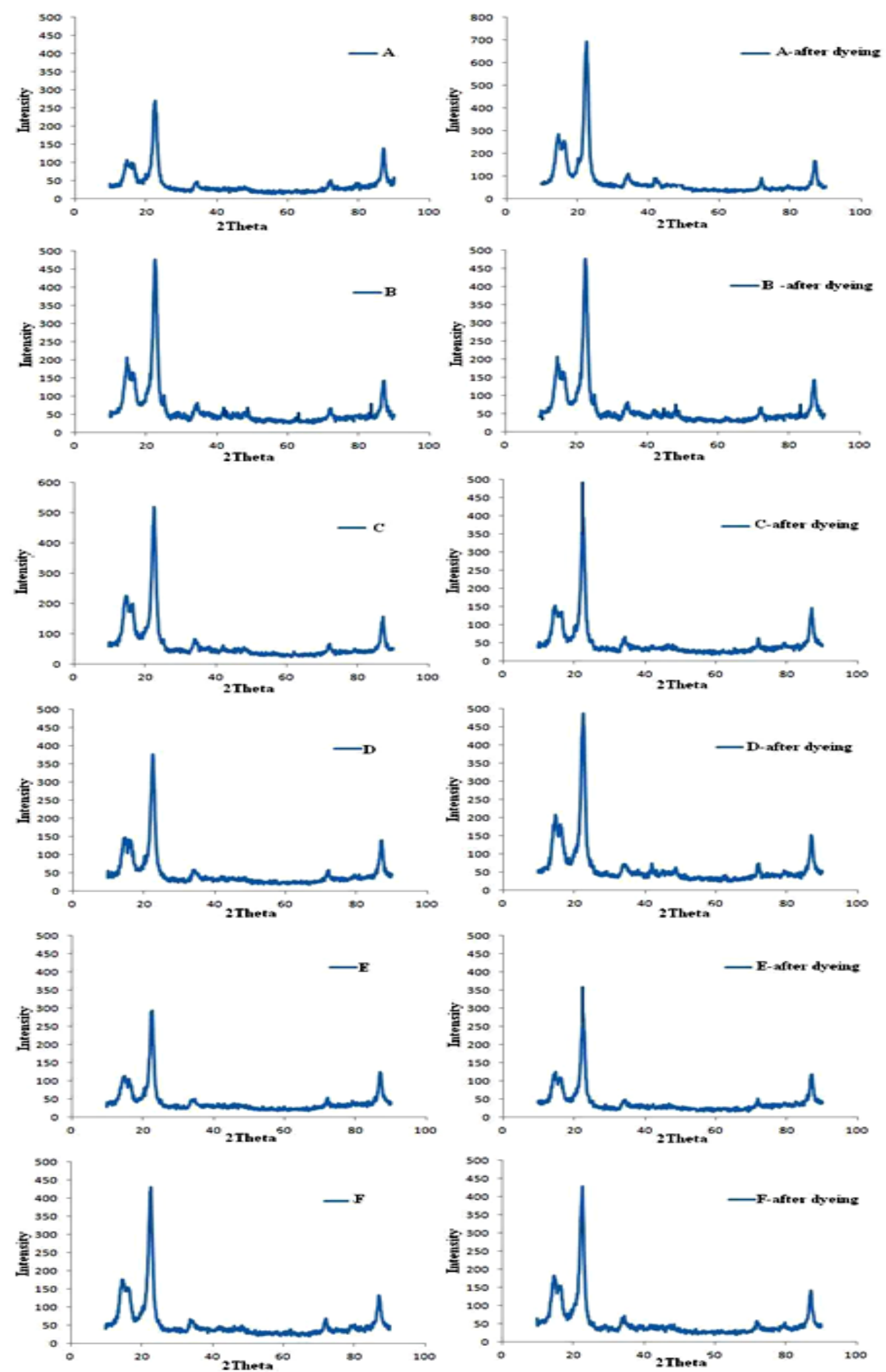

Figure 4. The XRD of treated and untreated samples before and after dyeing. 
It should be possible to determine accurately the percentage of crystallinity by comparing the intensity of diffracted X-ray. Intensity of the diffracted is shown in Figure 4. Peaks around 2 thetas of 14.7, 16.6 and 22.8 are associated with cellulose crystallite. As seen, by treating with BTCA/SHP, the crystallinity of the samples is decreased when comparing with untreated sample ( $F$ ). However by adding $\mathrm{TiO}_{2}$ nano-particles, the crystallinity of the sample is increased and some new peaks around 2 thetas of $25.3,36.8,37.84,48.0,53.9,55.1$ and 63 are attributed to $\mathrm{Ti}$ that appeared on the surface of Ti-loaded cotton samples. These peaks are more pronounced for $\mathrm{TiO}_{2}$ treated sample in absence of BTCA.

In case of $D$ and $E$ samples, it is observed that the intensity of peak around 2-theta of 22.8 has shown a decrease and the crystallinity of the samples is reduced. Generally, it can be concluded that the crystallinity might be increased by dyeing the samples. The result of XRD complies with the results achieved by water absorption time.
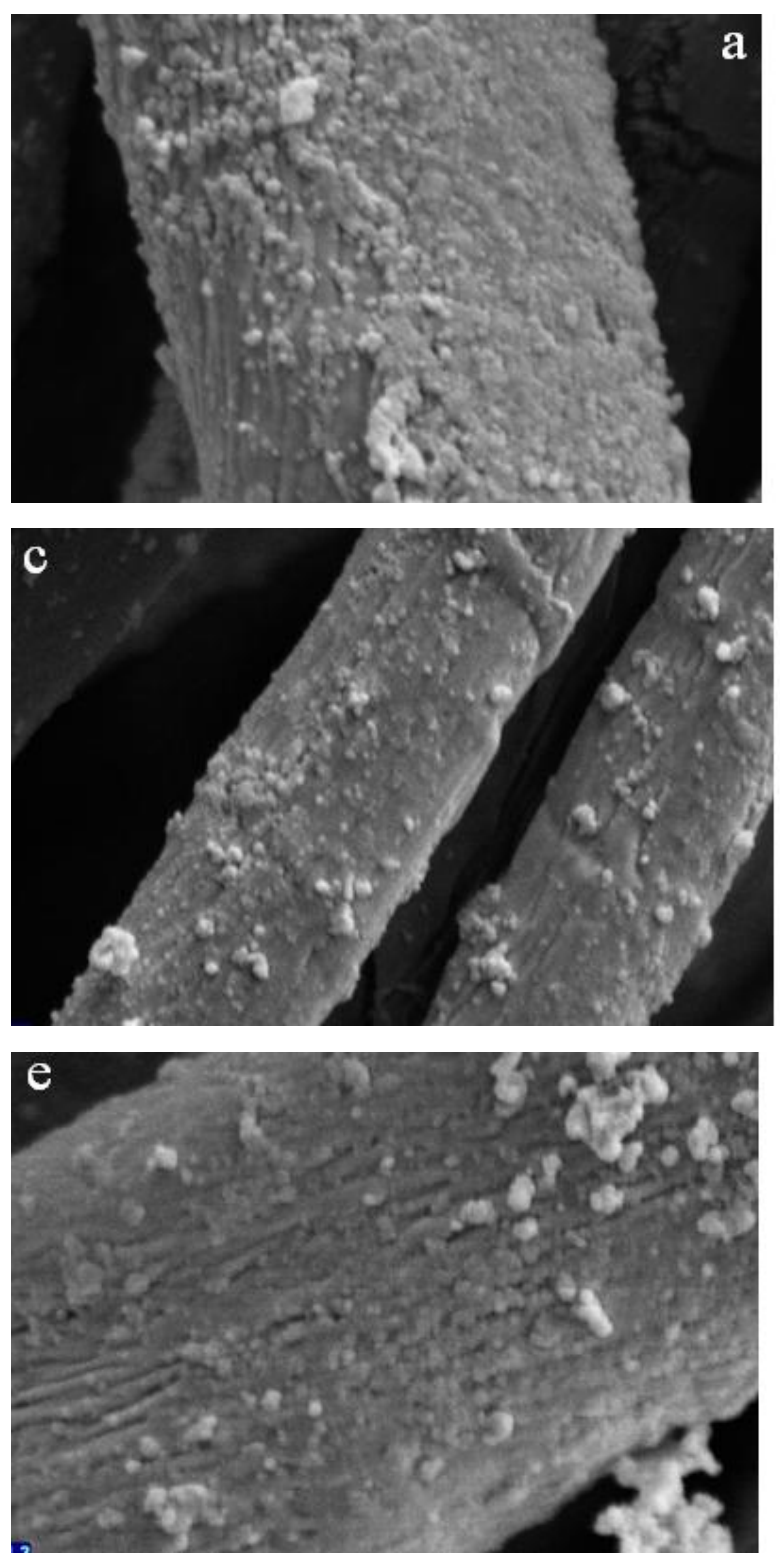

As it was mentioned before, direct dyeing causes increasing the water absorption time. It can be attributed to the crystallinity of the samples. By increasing the percentage of crystallinity, the penetration of water droplet through the surface of cotton fabrics becomes more complicated.

In case of $\mathrm{D}$ and $\mathrm{E}$, adding the $\mathrm{TiO}_{2}$ nano-particles increases the absorption time of water. This causes water repellent property on the surface due to blocking some of the hydrogen bonds of cellulosic chains. Therefore absorption time may become longer. In this case decreasing the crystallinity does not play a vital role.

\subsection{Microscopical evaluation}

SEM micrographs (Figure 5) of sample $A(B T C A+S H P)$, sample $\mathrm{B}\left(\mathrm{TiO}_{2} 2 \%\right)$, and sample $\mathrm{C}\left(\mathrm{BTCA}+\mathrm{SHP}>>>\mathrm{TiO}_{2}\right)$ show interesting results. In sample $A$ (Figure $5 \mathrm{a}$ and $5 \mathrm{~b}$ ), finishing agents fairly covered the surface of the samples even before dyeing. By the way, after dyeing, dyestuffs had better
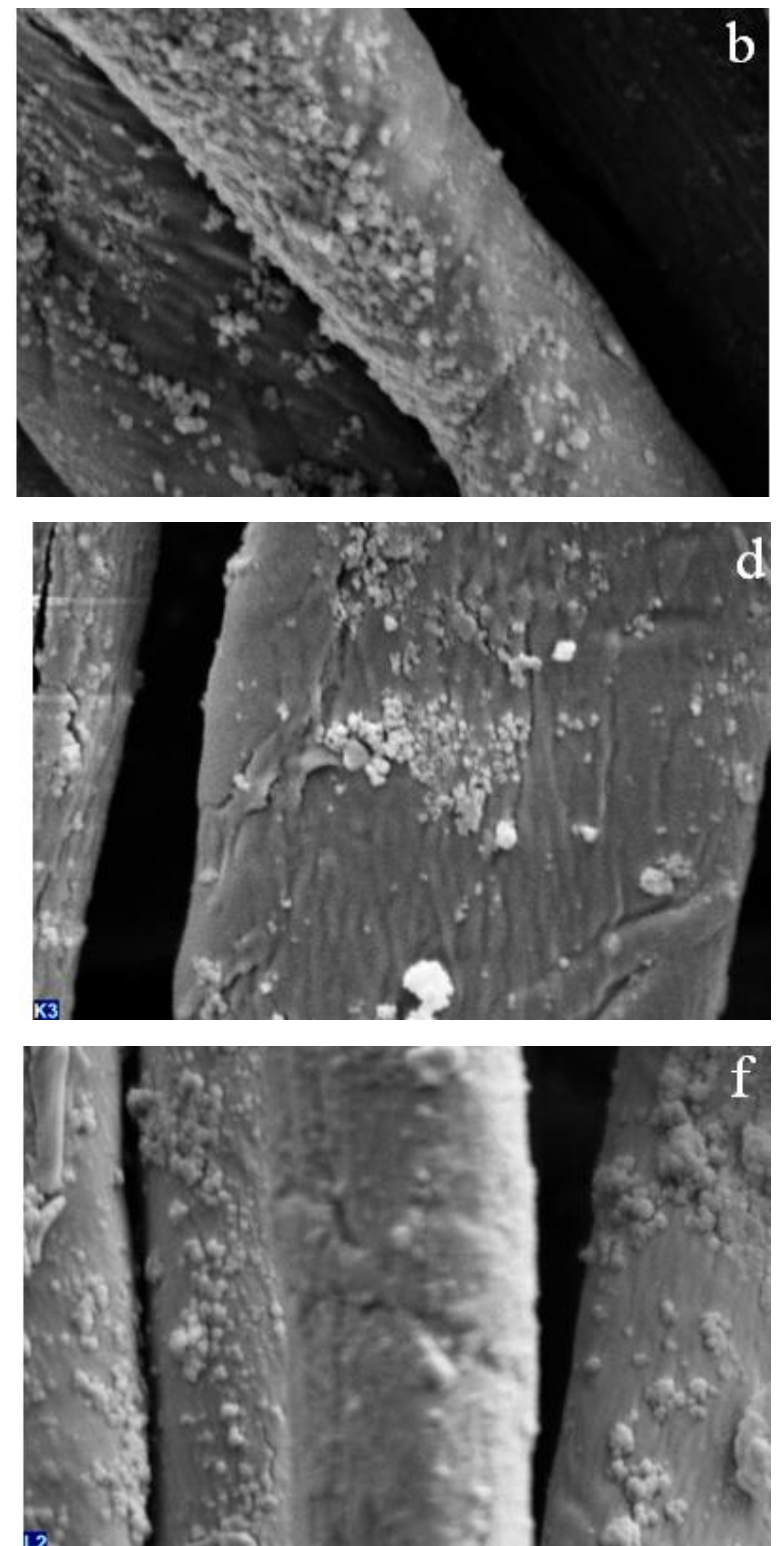

Figure 5. The SEM micrographs of treated samples; a) Sample.A before dyeing, b) Sample.A after dyeing; c) Sample.B before dyeing, d) Sample.B after dyeing; e) Sample.C before dyeing, f) Sample.C after dyeing. 
chances to react with treatment already laid on the surface. In other samples (Mainly in B) finished just by nano-particles, in some regions, particles have not been evenly distributed. There was no finishing process on these samples, just that they were treated by nano-particles. It can be inferred that finishing agents such as BTCA and SHP play an important role to hold particles in much more effective ways. According to the results reported in Table 2, not even satisfactory wrinkle resistant property was observed from samples which were finished only by nano-particles. This is in tight correlation with the results listed in Table 3. For samples finished only by nano-particles, wettability and dyeability illustrated less progressive trend. Therefore, finishing by agents before treating the samples by nano-particles enables samples to show higher wrinkle resistant property, moreover considerably better wettability and dyeability. Although there are seen some agglomeration of the particles on the surface in samples such as $\mathrm{C}$ finished by $\mathrm{TiO}_{2}$ nano-particles (Figure 5c-d), after treating by finishing agents and nano-particles, surfaces present different coverage which is in compliance with the conclusion made earlier.

\section{Conclusion}

The aim of this study was to investigate the wrinkle resistant property of cotton treated by BTCA, SHP in both presence and absence of nano- $\mathrm{TiO}_{2}$, before and after dyeing. Wettability of both untreated and cross-linked samples was evaluated using water drop test. The results show that adding nano- $\mathrm{TiO}_{2}$ raised the water absorption time. In addition, direct dyeing causes more increment in water absorption time. It could be concluded that the direct dye could not only be applied for cross-linked cotton dyeing with satisfactory dyeing properties but could also provide the cotton with excellent anti-wrinkle properties. The durability and fastness of dyed cross-linked samples were reported satisfactory and better as compared with untreated dyed sample.

\section{Acknowledgement}

Authors would like to express their gratitude from Islamic Azad University, Arak Branch for its financial support to run and complete this research project.

\section{References}

[1] Lee, J., Broughton, R.M., Akdag, A., Worle,y S.D., Huang, T.Sh. (2007). Antimicrobial fibers created via polycarboxylic acid durable press finishing. Textile Research Journal, 77(8), 604-611.

[2] Ibrahim, N.A., Refaie, R., Ahmed, A.F. (2010). Novel approach for attaining cotton fabric with multi-functional properties. Textile Research Journal, 40(1), 65-83.

[3] Sauperl, O., Stana-Kleinschek, K. (2010). Differences between cotton and viscose fibers crosslinked with BTCA. Textile Research Journal, 80(4), 383-392.

[4] Lam, Y.L., Kan, C.W., Yuen, C.W.M., Au, CH. (2011). Fabric objective measurement of the plasma-treated cotton fabric subjected to wrinkle-resistant finishing with BTCA and $\mathrm{TiO}_{2}$ system. Fibers and Polymers, 12(5), 626-634.

[5] Dang, N.Y., Ma, W., Zhang, Sh.F., Tang, B.T., Yang, J.Z. (2010). Studies on anti-wrinkle properties of silk fabrics dyed with reactive and cross-linking dyes. Textile Research Journal, 80(4), 374-382.

[6] Yang, Ch.Q., Lu, Y., Lickfield, G.C. (2002). Chemical analysis of 1,2,3,4-butanetetracarboxylic acid. Textile Research Journal, 72(9), 817-824.

[7] Yang, Ch.Q., Qian. L., Lickfield, G.C. (2001). Mechanical strength of durable press finished cotton fabric. Part IV: Abrasion resistance. Textile Research Journal, 71(6), 543548.

[8] Yang, Ch.Q., Wei, W., Lickfield, G.C. (2000). Mechanical strength of durable press finished cotton fabric: Part II: Comparison of cross-linking agents with different molecular structures and reactivity. Textile Research Journal, 70(2), 143-147.

[9] Nazari, A., Montazer, M., Rashidi, A., Yazdanshenas, M., Anary-Abbasinejad, M. (2009). Nano-TiO 2 photo-catalyst and sodium hypophosphite for cross-linking cotton with poly carboxylic acids under UV and high temperature. Applied Catalysis A: General, 371(1-2), 10-16.

[10] Paul, R., Bautista, L., Varga, M.D., Botet, J.M., Casals, E., Puntes, V., Marsal, F. (2010) Nano-cotton fabrics with high ultraviolet protection. Textile Research Journal, 80(52), 454-462.

[11] Hashemikia, S., Montazer, M. (2012). Sodium hypophosphite and nano $\mathrm{TiO}_{2}$ inorganic catalysts along with citric acid on textile producing multi-functional properties. Applied Catalysis A: General, 417- 418, 200208.

[12] Liu, X., Zhao, X., Li, B., Cao, C., Dong, Y., Ding, Ch., Chu, PK. (2008). UV-irradiation-induced bioactivity on $\mathrm{TiO}_{2}$ coatings with nanostructural surface. Acta Biomater, 4(3), 544-552.

[13]Liuxue, Zh., Xiulian, W., Peng, L., Zhixing, Su. (2008). Low temperature deposition of $\mathrm{TiO}_{2}$ thin films on polyvinyl alcohol fibers with photocatalytical and antibacterial activities. Applied Surface Science, 254(16), 1771-1774.

[14] Wang, H., Wu, Zh., Zhao, W., Guan, B. (2007). Photocatalytic oxidation of nitrogen oxides using $\mathrm{TiO}_{2}$ loading on woven glass fabric. Chemosphere, 66(1), 185190.

[15] Euvananont, C., Junin, C., Inpor, K., Limthongkul, P. Thanachayanon, C. (2008). $\mathrm{TiO}_{2}$ optical coating layers for self-cleaning applications. Ceramics International, 34(4), 1067-1071.

[16] Uddin, M.J., Cesano, F., Bonino, F., Bordiga, S., Spoto, G., Scarano, D., Zecchina, A. (2007) Photoactive $\mathrm{TiO}_{2}$ films on cellulose fibers: synthesis and characterization. Journal of Photochemistry and Photobiology A: Chemistry, 189(2-3), 286-294.

[17] Fujishima, A., Zhang, $X . \quad$ (2006). Titanium dioxide photocatalysis: present situation and future approaches. Comptes Rendus Chimie, 9(5-6), 750-760.

[18] Yuen, C.W.M., Ku, S.K.A., Kan, C.W, Cheng, Y.F, Choi, P.S.R, Lam, Y.L. (2007). Using nano-TiO ${ }_{2}$ as co-catalyst for improving wrinkle-resistant of cotton fabric. Surf. Rev. Lett, 14(4), 571-575.

[19] Wells, PB. (2001). Encyclopedia of Materials: Science and Technology, 1020-1025, Elsevier Science Ltd. 
[20] Lam, Y.L., Kan, C.W., Yuen, C.W.M. (2011). Wrinkleresistant finishing of cotton fabric with BTCA-the effect of co-catalyst. Textile Research Journal, 81(5), 482-493.

[21] Lam, Y.L., Kan, C.W., Yuen, C.W.M. (2010). Effect of concentration of titanium dioxide acting as catalyst or cocatalyst on the wrinkle-resistant finishing of cotton fabric. Fibers and Polymers, 11(4), 551-558.

[22] Yang, C., Liang, G.L., Xu, K.M., Gao, P., Xu, B. (2009), Bactericidal functionalization of wrinkle-free fabrics via covalently bonding TiO @ Ag nanoconjugates. J Mater Sci, 44(7), 1894-1901.

[23] Veronovski, N., Rudolf, A., Sfiligoj, S.M., Kreže, T., Geršak, J. (2009). Self-cleaning and handle properties of $\mathrm{TiO}_{2}$ modified textiles. Fibers and Polymers, 10(4), 551-556.
[24] Shaikhzadeh Najar, S., Hezavehi, E., Hoseini Hashemi, Sh., Rashidi, A. (2009). Investigation into wrinkle behavior of woven fabrics in a cylindrical form by measuring their tangential force. Int. J. Cloth. Sci. Tech, 21 (1), 7-30.

[25] Azad, A., Hezavehi, E., Shahvazian, M., Zolgharnein, P. (2012). Study of BTCA and Nano-TiO Effect on Wrinkle Force and Recovery of Cotton/Polyester Blended Fabric. Fibers \& Textiles in Eastern Europe, 20 5(94), 60-65.

[26] Hezavehi, E., Shaikhzadeh Najar, S., Zolgharnein, P., Yahya, $H$. (2011). A new electro-mechanical technique for measurement of stress relaxation of polyester blended fabric with constant torsional strain. Int. J. Cloth. Sci. Tech, 23 (5), 388-98. 